\title{
Effects of Preoperative Use of Oral Dextromethorphan on Postoperative Need for Analgesics in Patients With Knee Arthroscopy
}

\author{
Saeid Reza Entezary ${ }^{1}$, Saeedeh Farshadpour ${ }^{1, *}$, Mahmood Reza Alebouyeh ${ }^{1}$, Farnad Imani ${ }^{1}$, \\ Mohammad Kazem Emami Meybodi ${ }^{2}$, Habibollah Yaribeygi ${ }^{3}$ \\ ${ }_{2}^{1}$ Department of Anesthesiology, Rasoul Akram Medical Center, Iran University of Medical Sciences, Tehran, Iran \\ ${ }_{3}^{2}$ Department of Orthopedic, Faculty of Medicine, Baqiyatallah University of Medical Sciences, Tehran, Iran \\ ${ }^{3}$ Department of Physiology, Faculty of Medicine, Baqiyatallah University of Medical Sciences, Tehran, Iran \\ ${ }^{*}$ Corresponding author: Saeedeh Farshadpour, Department of Anesthesiology, Rasool-e-Akram Hospital, Iran University of Medical Sciences, Tehran, Iran. Tel: +98-9131511461, E-mail: \\ saeedeh_farshadpour@yahoo.com. \\ Received: March 12, 2013; Revised: June 25, 2013; Accepted: July 4, 2013
}

\begin{abstract}
Background: Studies have shown that N-methyl-D-aspartate receptor(NMIDA) plays an essential role in postoperative pain. It seems that use of NMDA receptor antagonists such as Dextromethorphan intensifies the analgesic effects of opioids.

Objectives: In this study, we evaluated the effect of preoperative administration of Dextromethorphan on postoperative pain reduction. Patients and Methods: This double blind randomized clinical trial was conducted on arthroscopic surgery candidates. Participants were randomly allocated to interventions and assigned to two groups of Dextromethorphan and placebo. In Dextromethorphan group, the patients received $1 \mathrm{mg} / \mathrm{kg}$ Dextromethorphan orally the night before the operation. Pain severity based on the visual analog scale (VAS) up to 16 hours postoperation, use of opioids, and the first request for analgesics were recorded postoperatively.

Results: A total of 112 patients in the Dextromethorphan $(n=54)$ and placebo groups $(n=58)$ were evaluated. No significant difference was detected between the two groups for age, sex or ASA. The mean amount of opioid consumption was significantly lower in patients who received Dextromethorphan $(10.7 \pm 5.6 \mathrm{mg})$ compared to the placebo group $(13.1 \pm 5.6 \mathrm{mg}),(\mathrm{P}=0.03)$. The mean time until the first opioid request in patients who received Dextromethorphan was longer than that in the placebo group $(\mathrm{P}=0.01)$.

Conclusions: The study results demonstrated that preemptive use of Dextromethorphan reduced postoperative pain and opioid consumption.
\end{abstract}

Keywords: Pain; Postoperative Period; Analgesics, Opioid; Analgesia

\section{Background}

Postoperative pain management is an important part of postoperative care and plays an important role in postsurgical ambulation and general status of patients (1-4). Also, perioperative pain can adversely affect patient's hemodynamic status, and increase the risk of several cardiovascular complications like myocardial infarction and cerebrovascular accidents such as stroke, and etc. (1, 2 ). Some studies have shown different effects of various forms of a drug on pain relief (5-7). Peri- and postoperative pain can lead to central sensitization and subsequent increased perception of pain by patients. This converts acute postoperative pains to chronic pains per se (1). Some methods have been proposed to decrease pain after surgery (8). Elimination of perioperative pain by using opioids is a common method which is followed by their adverse side effects (2). To decrease these complications, other nonopioid medications can be used to relieve pain before the operation (9-13) or after it $(14,15)$. Peripheral tissue damage and subsequent inflammatory response cause a local change in sensitivity of peripheral receptors and increase the excitability of neurons present in the spinal cord $(2,4)$. Studies have demonstrated that $\mathrm{N}$-methyl-D-aspartate receptors (NMDA) play a part in increasing the sensitivity of spinal receptors (15). Therefore, considering this mechanism, a preventive method namely preemptive analgesia has been used to decrease postoperative pain recently (6). Furthermore, concomitant use of NMDA receptor antagonists and opioids can enhance the analgesic effects of opioids and stop tolerance to the analgesic action of opioids.

At present, there is a possibility that preemptive analgesia with NMDA receptor antagonists decreases postoperative pain of patients. Dextromethorphan, ketamine, amantadine, and magnesium sulfate are among the wellknown NMDA receptor antagonists. Dextromethorphan and its metabolites are considered as noncompetitive low affinity NMDA receptor antagonists (15). Considering the fact that Dextromethorphan is a widely available oral medication and has minimal side effects when adminis-

Implication for health policy/practice/research/medical education:

Opioid administration must be limited in patients due to their severe side effects. We showed that administration of Dextromethorphan can cause a reduction in consumption of opioid analgesics compared to the placebo after arthroscopic knee surgery.

Copyright (C) 2013, Iranian Society of Regional Anesthesia and Pain Medicine(ISRAPM); Published by Kowsar Corp. This is an open-access article distributed under the terms of the Creative Commons Attribution License, which permits unrestricted use, distribution, and reproduction in any medium, provided the original work is properly cited. 
tered in low dosage, it has been used for pain control in various studies.

Several researchers have evaluated the analgesic effects of Dextromethorphan and its impact on peri-and postoperative need for analgesics (15). In one study it was demonstrated that administration of Dextromethorphan prior to abdominal surgery decreased the need for postoperative opioids significantly compared to the placebo group (9). Also, Dextromethorphan has been successfully used to relieve postoperative pain following mastectomy, hysterectomy, and diagnostic laparoscopy $(8,10)$. Considering the relevant literature, it seems that Dextromethorphan is effective in decreasing postoperative pain in patients and reduces administration of opioids. However, its preoperative use has yet to be thoroughly investigated. Further research is required to study the preoperative administration of Dextromethorphan in different groups of patients and various surgical procedures.

\section{Objectives}

Considering the effects of Dextromethorphan on decreasing the need for opioids and subsequent reduction in their side effects, causing preemptive analgesia and less need for administration of perioperative analgesics to prevent central sensitization and reducing the incidence of chronic pain, the present study aimed to compare the effect of Dextromethorphan and placebo on reducing the need for administration of opioids and subsequent reduction in their potential side effects.

\section{Patients and Methods}

In this randomized double blind controlled clinical trial, candidates for arthroscopic knee surgery admitted to the Orthopedic Department of Rasoul-e-Akram Hospital during 2008-2009 were recruited using convenience sampling. The inclusion criteria were as follows:

- Being a candidate for arthroscopic knee surgery

- Spinal anesthesia

- Older than 18 years

- ASA classes I and II

The exclusion criteria were:

- Obese patients

- Not consenting to spinal anesthesia

- Conversion of arthroscopic knee surgery to open knee surgery

- History of opioid consumption

- Use of sedatives or analgesics peri operatively

After patient selection based on the inclusion and exclusion criteria, they were assigned to the Dextromethorphan and placebo groups alternately. All patients were visited the night before the operation and signed a written informed consent. Instructions were given on how to use the VAS ruler to measure subjective complaints of pain, and the whole procedure was thoroughly explained to the subjects. In the Dextromethorphan group, patients consumed oral Dextromethorphan syrup $1 \mathrm{mg} / \mathrm{kg}$ 2-3 hours before the operation (due to its short half-life). The placebo group consumed normal saline. Also, all patients took a $10 \mathrm{mg}$ Diazepam tablet. In the operating room, an IV line was taken and $500 \mathrm{cc}$ of IV fluid was administered. Patients were placed in a seated position while being monitored, and Marcaine $0.5 \%$ was injected as the analgesic agent (per body weight and about 3-3.5 mL at all) into the epidural space at L4/L5 with a 25 gauge needle. Patient's position was then quickly changed to supine and blood pressure was monitored. After completion of the operation, patients were transferred to the recovery room and then to the ward. The time of the first opioid request after the operation, and the intensity of pain at different time points were recorded.

Demographic characteristics of patients including age, sex, surgical indication, and etc. were extracted from their medical files or asked directly and recorded in data collection sheets. Severity of pain in patients was measured 4, 8, 12, 16 and 24 hours postoperatively using the visual analog scale. The patients were received analgesic drug (Morphine) every four hours or PRN when needed. The time of first opioid request after the operation was also recorded as an indicator of postoperative pain severity from the moment of entering the recovery room to patient's first complaint of pain in minutes. Eventually, the total amount of Morphine administered within 24 hours was evaluated as an indicator of pain intensity.

The obtained data were statistically analyzed using SPSS version 13 software. Quantitative data were represented as mean and standard deviation, and qualitative data as frequency. To compare the qualitative and quantitative data between the two groups, in case of normal distribution of data, chi square test and t-test were used, respectively. If data were not normally distributed, nonparametric statistical tests were used. Also repeated measurements ANOVA was used to evaluate the effect of time and its correlation with $\operatorname{drug}(\mathrm{P}<0.05$ was considered statistically significant).

\section{Results}

A total of 112 patients in the two groups of Dextromethorphan $(n=54)$ and placebo $(n=58)$ were evaluated. No side effects about Dextromethorphan administration in these patients were reported. Table 1 summarizes the demographic and clinical characteristics of patients. As observed, no statistically significant difference existed between the two groups for age, gender or ASA status.

Table 2 shows the severity of pain during the first 16 hours after the operation. As observed, the mean score of pain during the first 8 hours after the operation in the 
Entezary SR et al.

\begin{tabular}{|c|c|c|c|}
\hline & $\begin{array}{l}\text { Dextromethorphan } \\
(\mathrm{n}=54)\end{array}$ & $\operatorname{Placebo}(n=58)$ & Pvalue \\
\hline Age, mean (SD), y & $28.1 \pm 8.6$ & $30.2 \pm 7.5$ & 0.1 \\
\hline Frequency of males, \% & $(66.7) 36$ & $(63.8) 37$ & 0.7 \\
\hline \multicolumn{4}{|l|}{ ASA status, $\%^{\mathrm{a}}$} \\
\hline ASA I & $(70.4) 38$ & (77.6) 45 & 0.3 \\
\hline ASA II & $(29.6) 16$ & $(22.4) 13$ & 0.3 \\
\hline
\end{tabular}

${ }^{a}$ Abberviations: ASA, acetylsalicylic acid.

Table 2. Severity of Postoperative Pain in the Two Groups

\begin{tabular}{llll}
\hline & Dextromethorphan Group $(\mathbf{n}=\mathbf{5 4})^{\mathrm{a}}$ & ${\text { Placebo Group }(\mathbf{n}=\mathbf{5 8})^{\mathrm{a}}}$ & Pvalue \\
\hline In the recovery room & $4.1 \pm 1.8$ & $4.7 \pm 1.2$ & 0.05 \\
\hline 4 hours postoperation & $2.9 \pm 0.9$ & $3.8 \pm 1.1$ & 0.001 \\
8 hours postoperation & $2.7 \pm 0.7$ & $3.4 \pm 0.6$ & 0.001 \\
$\mathbf{1 2}$ hours postoperation & $2.4 \pm 0.6$ & $2.5 \pm 0.7$ & 0.381 \\
\hline $\mathbf{1 6}$ hours postoperation & $0.9 \pm 0.6$ & $1.2 \pm 0.7$ & 0.09 \\
\hline
\end{tabular}

$\mathrm{a}$ Mean $+\mathrm{SD}$

Table 3. Severity of Pain After the Operation in the Two Groups

\begin{tabular}{|c|c|c|c|}
\hline & Dextromethorphan $(n=54)$ & $\operatorname{Placebo}(\mathbf{n}=\mathbf{5 8})$ & P value \\
\hline Opioid consumption, mg, Mean \pm SD & $10.7 \pm 5.6^{\mathrm{a}}$ & $13.1 \pm 6.1^{\mathrm{a}}$ & 0.03 \\
\hline $\begin{array}{l}\text { Time of the first analgesic postopera- } \\
\text { tion, , mean } \pm \text { SD, hours }\end{array}$ & $3.7 \pm 1.3^{\mathrm{a}}$ & $3.1 \pm 1.2^{\mathrm{a}}$ & 0.01 \\
\hline \multicolumn{4}{|l|}{ Frequency of side effects of opioids ,\% } \\
\hline Nausea and vomiting, \% & $(24.1) 13$ & (39.7) 23 & 0.08 \\
\hline Itching, \% & (18.5) 10 & $(20.7) 12$ & 0.7 \\
\hline
\end{tabular}

${ }^{\mathrm{a}}$ Mean $\pm \mathrm{SD}$

Dextromethorphan group was less than that in the placebo group. During the first 12 and 16 hours postoperatively, the mean score of pain in the test group was also lower than the placebo group, but this difference was not statistically significant. According to the Repeated Measures ANOVA, the decreasing trend of pain severity in the Dextromethorphan group was significantly greater than the placebo group $(\mathrm{P}=0.001)$. Time factor also played a part in this respect, and a part of pain reduction was attributed to the passing of time $(\mathrm{P}=0.001)$. The correlation between premedication and time was not statistically significant $(\mathrm{P}=0.121)$.

Administration of opioids as analgesics during the first 24 hours postoperation is demonstrated in Table 3 . The mean amount of opioid consumption was $10.7 \pm 5.6 \mathrm{mg}$ in the Dextromethorphan group, which was significantly lower than the mean rate of consumption in the placebo group (13.1 $\pm 6.1 \mathrm{mg}),(\mathrm{P}=0.032)$. The mean time until the first opioid request after the operation was $3.7 \pm 1.3$ hours in the Dextromethorphan group, which was significantly longer than the time in the placebo group $(\mathrm{P}=0.011)$ (Table 3). For occurrence of side effects due to the adminis- tration of opioids, 13 (24.1\%) subjects in the Dextromethorphan and 23 (39.7\%) in the placebo group experienced nausea and vomiting. Although the prevalence of these complications in the placebo group was greater than the test group, this difference was not statistically significant $(P=0.08)$. Incidence of itching was not significantly different between the two groups of Dextromethorphan (18.5\%) and placebo (20.7\%), ( $\mathrm{P}=0.773)$.

\section{Discussion}

This study showed that preemptive oral Dextromethorphan can reduce the need for administration of opioids after arthroscopic knee surgery compared to the placebo. Also, it was demonstrated that the need for first analgesic was significantly later in the test group compared to the placebo group. As mentioned earlier, postoperative pain is a serious problem in surgical wards and plays a critical role in patient's postoperative general status and their discharge time from the hospital $(1,2)$. In contrast to what was previously believed, assessment of pain reduction interventions by measuring pain severity at dif- 
ferent time points cannot be a suitable indicator for their effectiveness (1) because ethically, analgesics have to be administered for patients who experience severe pains (i.e. VAS > 3). Therefore, the main key in studies conducted on control and management of postoperative pain is to evaluate the total consumption of opioids and reduced consumption in a group of patients is indicative of the effectiveness of that specific intervention (1). As mentioned earlier, the changes in pain severity at different time points were similar in the both groups, but the rate of opioid consumption was significantly lower in the test group compared to the placebo. During the recent years, Dextromethorphan has been used as an $\mathrm{N}$-methyl-d-aspartate receptor channel blocker by the action of its metabolite Dextrophan (6). Recent studies have shown that NMDA receptor plays a part in increasing the central sensitization. Therefore, we can decrease postoperative pain by blocking this receptor before the operation using preemptive methods (5). Furthermore, it seems that simultaneous administration of NMDA receptor antagonists and opioids can enhance the analgesic effect of opioids and stop tolerance to analgesic effects of opioids (7). In a study in 1999, it was demonstrated that preemptive Dextromethorphan decreased pain severity and amount of Morphine administration significantly after the operation which is in accordance with our findings. Furthermore, other findings regarding this subject confirm the effectiveness of Dextromethorphan. This is in accordance with physicians' intentions to decrease the administration of opioids.

Although reduced consumption of opioids leads to a reduction in their potential side effects like nausea, vomiting and itching, and also in the present study, incidence of these complications was somehow lower in the test group, this difference was not statistically meaningful.

In general, it appears that Dextromethorphan can be effective to reduce postoperative pain as a safe drug available over the counter (OTC) if administered preemptively. However, to generalize the results to other surgical procedures, further investigations are required.

The present study results revealed that administration of Dextromethorphan can reduce consumption of opioid analgesics compared to the placebo after arthroscopic knee surgery. However, this study failed to significantly reduce the incidence of opioid side effects by preoperative consumption of Dextromethorphan in comparison to the placebo.

\section{Acknowledgements}

The authors would like to thank the collaboration of Department of Anesthesiology, Iran University of Medical Sciences.

\section{Authors' Contribution}

Saeid Reza Entezary, Mahmood Reza Alebouyeh, and Farnad Imani developed the original idea and supervised throughout the study. Saeedeh farshadpour and Mohammad kazem Emami meybodi developed the protocol, collected the data, and wrote the abstract and manuscript, and is guarantor. Habibollah Yaribeygi edited and revised the manuscript body.

\section{Financial Disclosure}

This study had not any financial disclosure and or any funding support.

\section{Funding/Support}

This study had not any financial disclosure and or any funding support.

\section{References}

1. Imani F. Postoperative pain management. Anesth Pain. 2011;1(1):67.

2. Imani F, Safari S. Pain Relief is an Essential Human Right”, We Should be Concerned about It. Anesth Pain. 2011;1(2):55-7.

3. Shoar S, Esmaeili S, Safari S. Pain Management After Surgery: A Brief Review. Anesth Pain. 2012;1(3):184-6.

4. Lee RM, Tey JBL, Chua NHL. Postoperative Pain Control for Total Knee Arthroplasty: Continuous Femoral Nerve Block Versus Intravenous Patient Controlled Analgesia. Anesth Pain. 2012;1(4):239-42.

5. Kverma R, Paswan A, De A, Gupta S. Premedication with Midazolam Nasal Spray: An Alternative to Oral Midazolam in Children. Anesth Pain . 2012;1(4):248-251.

6. Moradi M, Esmaeili S, Shoar S, Safari S. Use of Oxycodone in Pain Management. Anesth Pain. 2012;1(4):262-4.

7. Alimian M, Imani F, Faiz SHR, Pournajafian A, Navadegi SF, Safari S. Effect of Oral Pregabalin Premedication on Post-Operative Pain in Laparoscopic Gastric Bypass Surgery. Anesth Pain. 2012;2(1):126.

8. Amiri HR, Safari S, Makarem J, Rahimi M, Jahanshahi B. Comparison of Combined Femoral Nerve Block and Spinal Anesthesia With Lumbar Plexus Block for Postoperative Analgesia in Intertrochanteric Fracture Surgery. Anesth Pain. 2012;2(1):32-5.

9. Hosseini JSA, Hosseini VSM, Hatamian S. Comparison Between Effect Of Lidocaine, Morphine And Ketamine Spray On Post-Tonsillectomy Pain In Children. Anesth Pain. 2012;2(1):17-21.

10. Jazayeri SM, Mosaffa F, Abbasian MR, Reza H. Comparing the Efficacy of Intra-Articular Application of Morphine and Tramadol on Postoperative Pain After Arthroscopic Knee Surgery. Anesth Pain. 2012;2(1):28-31.

11. Imani F, Rahimzadeh P. Gabapentinoids: Gabapentin and Pregabalin for Postoperative Pain Management. Anesth Pain. 2012;2(2):52-53.

12. Alimian M, Imani F, Hassani V, Rahimzadeh P, Sharifian M, Safari S. Effects of Single-Dose Pregabalin on Postoperative Pain in Dacryocystorhinostomy Surgery. Anesth Pain . 2012;2(2):72-76.

13. Moghaddam MJ, Ommi D, Mirkheshti A, Dabbagh A, Memary E, Sadeghi A, et al. Effects of Clonidine Premedication Upon Postoperative Shivering and Recovery Time in Patients With and Without Opium Addiction After Elective Leg Fracture Surgeries. Anesth Pain. 2013;2(2):77-80.

14. Khahi MP, Marashi S, Khajavi MR, Najafi A, Yaghooti A, Imani F Postoperative Gabapentin to Prevent Postoperative Pain: A Randomized Clinical Trial. Anesth Pain. 2012;2(2):85-9.

15. Javid MJ, Hajijafari M, Hajipour A, Makarem J, Khzaeipour Z. Evaluation of A Low Dose Ketamine in Post Tonsillectomy Pain Relief: A Randomized Trial Comparing Intravenous and Subcutaneous Ketamine in Pediatrics. Anesth Pain. 2012;2(3):107-10. 